\title{
Uncommon True Isolated Macrodontia of a Maxillary Tooth
}

\author{
${ }^{1}$ Luciana Pereira, ${ }^{2}$ Priscila de Almeida Assunção, ${ }^{3}$ Sabrina Loren de Almeida Salazar, ${ }^{4}$ Fábio Ribeiro Guedes \\ ${ }^{5}$ Aline Corrêa Abrahão, ${ }^{6}$ Márcia Grillo Cabral, ${ }^{7}$ Lucianne Cople Maia
}

\begin{abstract}
Macrodontia is a rare dental anomaly which consists on the presence of any tooth or teeth larger than the average. In the present report the case of a child with an uncommon true isolated macrodontia of a maxillary central incisor is presented. The maxillary left central incisor was considered a macrodontic tooth since its mesiodistal crown dimension was $12.41 \mathrm{~mm}$ while the mesiodistal crown dimension of the maxillary right central incisor was $10.06 \mathrm{~mm}$. The radiographic examination confirmed an enlarged maxillary left central incisor and a normal developing dentition. The patient did not report esthetic complaints, however, due the presence of anterior open bite and crowding, he was referred to orthodontic clinic. Thus, it must be point out the importance of the radiographic diagnosis to support the clinical findings, helping to plan and provide better treatment for the patient.
\end{abstract}

Keywords: Tooth abnormalities, Dentition, Permanent.

How to cite this article: Pereira L, de Almeida Assunção $P$, de Almeida Salazar SL, Guedes FR, Abrahão AC, Cabral MG, Maia LC. Uncommon True Isolated Macrodontia of a Maxillary Tooth. J Contemp Dent Pract 2014;15(1):116-118.

Source of support: Nil

Conflict of interest: None

\section{INTRODUCTION}

Macrodontia is the presence of any tooth or teeth larger than average, and is a rare dental anomaly, ${ }^{1}$ it may be categorized as true generalized or isolated. ${ }^{2}$ A tooth with a normal crown size in a small gnathic bone, previously called relative macrodontia, should not be included. ${ }^{1-3}$ Isolated macrodontia has been reported in literature only rarely and occasionally it may be associated with morphological

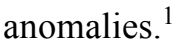

\footnotetext{
${ }^{1-3}$ Postgraduate Student, ${ }^{4,5}$ Adjunct Professor

${ }^{6}$ Associate Professor, ${ }^{7}$ Full Professor

${ }^{1-3,7}$ Department of Pediatric Dentistry and Orthodontics, School of Dentistry, Federal University of Rio de Janeiro, RJ, Brazil

${ }^{4-6}$ Department of Pathology and Oral Diagnosis, School of Dentistry, Federal University of Rio de Janeiro, RJ, Brazil
}

Corresponding Author: Lucianne Cople Maia, Professor Department of Pediatric Dentistry and Orthodontics, School of Dentistry, Federal University of Rio de Janeiro, RJ, Brazil Caixa Postal: 68.066, CCS-Cidade Universitária, Rio de Janeiro-RJ, Brazil, CEP: 21941-971, Phone: 021-21-25622101 e-mail: rorefa@terra.com.br
The etiology of macrodontia is unknown, and the prevalence varies between 0.5 and $2.5 \%{ }^{4}$ It appears as a dominant trait in some families. ${ }^{3,4}$ Isolated macrodontia may affect premolars, permanent maxillary, mandibular incisors, and mandibular third molars in descending order, and there is a higher prevalence in men than women. ${ }^{1,2}$

Macrodontia of anterior teeth causes problems of crowding, esthetics and plaque accumulation because of surface notching, and also additional problems of tooth interdigitation and overjet reduction. ${ }^{2}$ Treatment is not necessary, unless for esthetic reasons, which requires special restorative and orthodontic treatment planning. ${ }^{4}$

This report presents the case of a child with an uncommon true isolated macrodontia of a maxillary central incisor.

\section{CASE REPORT}

A 16-year-old boy attended to the Center of Surveillance and Monitoring of Dental-alveolus Injuries at the Federal University of Rio de Janeiro, Brazil, for a routine dental treatment. The medical history was not contributory. The patient's family history was investigated and did not disclose any hereditary anomalies, dental or otherwise. The intraoral examination revealed a mixed dentition period showing anterior open bite and crowding, which required orthodontic treatment for malocclusion, a fractured composite resin restoration of the maxillary right central incisor, and macrodontia of the maxillary left central incisor (Figs 1A to C). The radiograph examination showed an enlarged maxillary left central incisor and a normal developing dentition (Figs $2 \mathrm{~A}$ and $\mathrm{B}$ ), confirming the diagnosis of a single tooth macrodontia. Other dental anomalies were absent in the present case.

The patient is being followed up for clinical and radiographic evaluation. The orthodontic require for anterior open bite, crowding and malocclusion will be carried out.

\section{DISCUSSION}

The normal mesiodistal crown dimension of permanent maxillary central incisors is around $9.00 \mathrm{~mm} .{ }^{4,5}$ The patient in the present case showed a mesiodistal crown dimension of the maxillary right central incisor of around $10.06 \mathrm{~mm}$ and the maxillary left central incisor of around $12.41 \mathrm{~mm}$, which was considered a macrodontic tooth.

Up to now there are only seven reported cases of isolated macrodontia in the literature, and only one involving the 

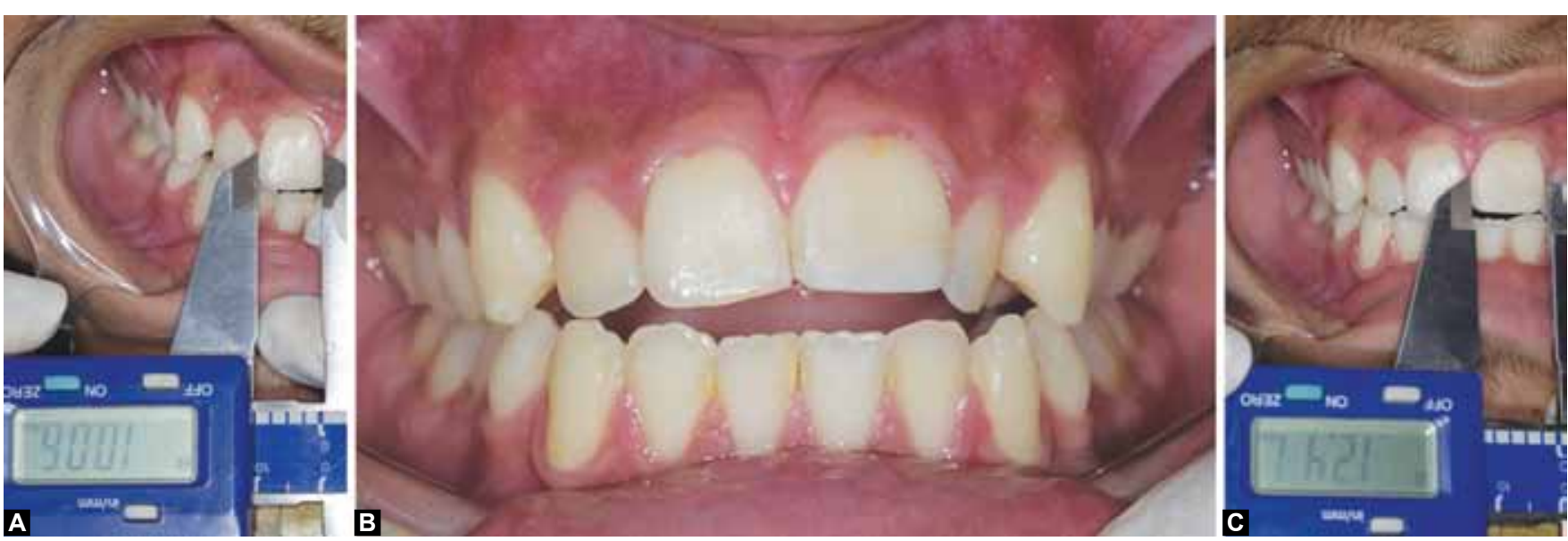

Figs 1 A to C: Intraoral examination showing macrodontia of the maxillary left central incisor, measured by digital caliper Mitutoyo Ltda., Sul Americana, Brazil (0-8 pol/0-200 mm); the normal mesiodistal crown dimension of permanent maxillary central incisor is around $9.00 \mathrm{~mm}$
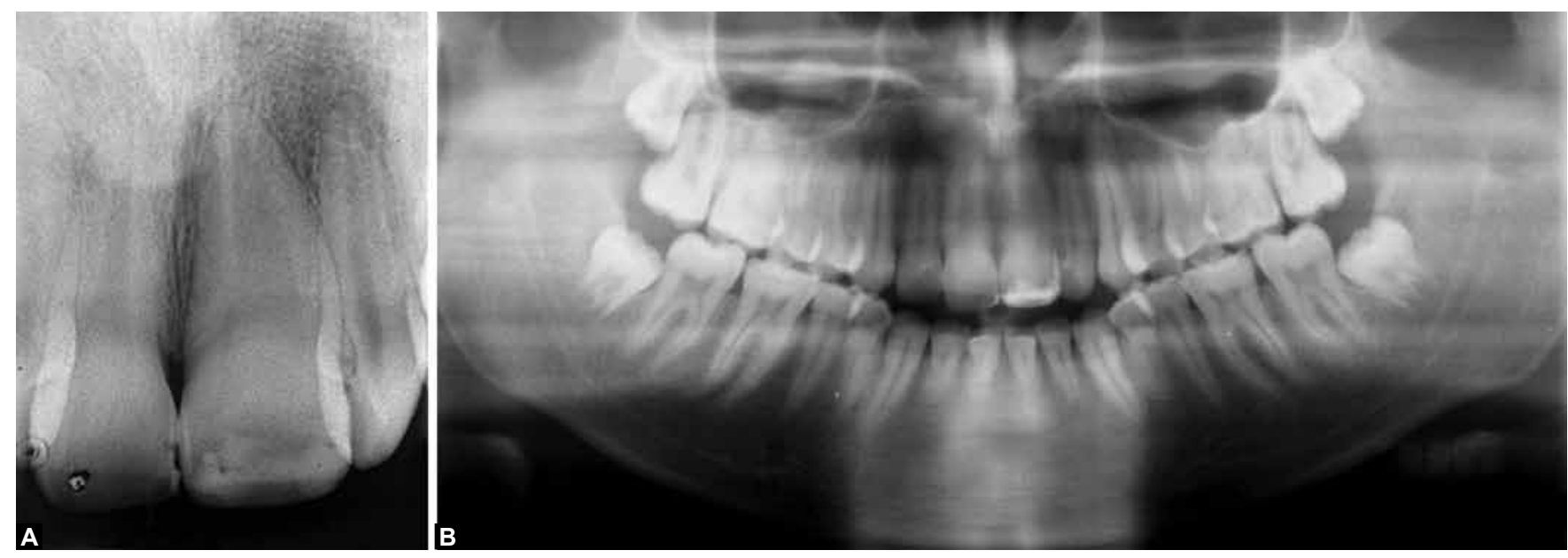

Figs 2A and B: Periapical and panoramic radiographs showed composite resin restoration of fractured maxillary right central incisor, subsequently replaced, and an enlarged maxillary left central incisor

maxilla. ${ }^{1}$ Although the etiology of macrodontia is unknown, genetic and environmental causes have been proposed such as pituitary gigantism, insulin-resistant diabetes, pineal hyperplasia, otodental syndrome, XYY males and unilateral facial hyperplasia. ${ }^{3}$ The Elkman-Westborg-Julin trait ${ }^{4}$ was discarded as there was no association of macrodontia with multituberculism. However, single-conical molar roots and the presence of grooves on the tooth surface were observed in our patient. Additionally, no family history could be established in the present case.

As part of a routine examination at the Center for Surveillance and Monitoring of Dental-alveolus Injuries of the Federal University of Rio de Janeiro, after clinical examination a periapical radiograph was taken, revealing an isolated macrodontia of the maxillary left central incisor. This was an uncommon observation in our routine practice, and could be considered a rare event in our patients profile. ${ }^{1,3}$

The change in the morphology of this tooth and also the concurring malocclusion problem may compromise the quality of the oral hygiene in a patient. ${ }^{4}$ Although the patient did not report esthetic complaints, the presence of anterior open bite and crowding led to his referral to orthodontic clinic where the treatment and maintaining of an ideal occlusion will be carried out.

\section{CONCLUSION}

An uncommon case of a true isolated macrodontia of a maxillary central incisor was reported here and in spite of unknown etiology, multifactorial, combining polygenetic and environmental influences should not be discarded. The etiology of macrodontia could become clearer if more cases were reported in the future. Here we also point out the importance of the radiographic diagnosis to support the clinical findings, to help plan and provide better treatment for patient care.

\section{BULLET POINTS}

\section{What This Paper Adds}

- The literature search revealed seven reported cases of true isolated macrodontia, and only one involving the maxilla. 
- The macrodontia with unilateral eruption is not rare in cases of unilateral facial hyperplasia.

- This paper contributes by reporting a further case of an uncommon true isolated macrodontia of maxillary central incisor, because it was not associated to unilateral facial hyperplasia.

\section{Why this Paper is Important for Pediatric Dentists}

- The importance of radiographic diagnosis, after clinical examination, to confirm the dental finding, and to help provide better patient care.

- The change in the morphology of the tooth and also the concurring malocclusion problem may compromise the quality of the oral hygiene of the patient, reflecting on quality of life; therefore special attention should be taken.

\section{REFERENCES}

1. Dugmore CR. Bilateral macrodontia of mandibular second premolars: a case report. Int J Paediatr Dent 2001;11(1): 69-73.

2. Groper JN. Macrodontia of a single tooth: review of literature and report of case. J Am Dent Assoc 1987;114(1):69.

3. Rootkin-Gray VF, Sheehy EC. Macrodontia of a mandibular second premolar: a case report. ASDC J Dent Child 2001; 68(5-6):347-349.

4. Peker I, Kayaoglu G. A case of Ekman-Westborg-Julin trait: endodontic treatment of a macrodontic incisor. Oral Surg Oral Med Oral Pathol Oral Radiol Endod 2009;107(5):e89-92.

5. Ooshima T, Ishida R, Mishima K, Sobue S. The prevalence of developmental anomalies of teeth and their association with tooth size in the primary and permanent dentitions of 1650 Japanese children. Int J Paediatr Dent 1996;6(2):87-94. 